\title{
Corrupção e Economia: análise da influência do índice de percepção da corrupção sobre o crescimento econômico, a dívida pública e a taxa de desemprego
}

\author{
Ian Vinicius Farias Rodrigues ${ }^{1}$ \\ Aline Tatiane Fank Jung ${ }^{2}$ \\ Max Nunes Murtinho ${ }^{3}$
}

\begin{abstract}
RESUMO
A corrupção não é um problema apenas no Brasil. Trata-se de um problema que atinge números elevados no mundo todo. Mesmo em países desenvolvidos a corrupção está presente. Como a corrupção política implica no uso indevido dos recursos públicos, é razoável supor que contribua para a ineficiência econômica. Diante disso, o artigo tem como objetivo analisar se há relação de causalidade entre a corrupção brasileira e o crescimento econômico, a dívida pública e a taxa de desemprego, nos períodos entre 2008 e 2018. Para isso, foram realizadas análises estatísticas de correlação. Como proxy de corrupção utilizou-se o Índice de Percepção da Corrupção IPC e os dados de cadastros irregulares do Tribunal de Contas da União. A hipótese da pesquisa é a de que o aumento da corrupção impacta negativamente no crescimento econômico, dívida pública e na taxa de desemprego. Os resultados dos testes estatísticos demonstraram não haver correlação entre as variáveis analisadas e, portanto, rejeita-se a hipótese de causalidade a corrupção e as variáveis econômicas.
\end{abstract}

Palavras-Chave: Corrupção; Crescimento Econômico; Dívida Pública; Desemprego.

Corruption and Economics: Analysis of the Influence of the Corruption Perception Index on Economic Growth, Public Debt and Unemployment Rate

\begin{abstract}
Corruption is not just a problem in Brazil. This is a problem that reaches high numbers worldwide. Even in developed countries corruption is present. As political corruption implies the misuse of public resources, it is reasonable to assume that it contributes to economic inefficiency. Given this, the article aims to analyze whether there is a causal relationship between Brazilian corruption and economic growth, public debt and unemployment rate, in the periods between 2008 and 2018. For this, correlation statistical analyzes were performed. As a proxy for corruption, the Corruption Perception Index (IPC) and data from irregular records of the Federal Court of Auditors were used. The research hypothesis is that the increase in corruption negatively impacts economic growth, public debt and unemployment rate. The results of the statistical tests showed no correlation between the variables analyzed and, therefore, the hypothesis of causality, corruption and economic variables is rejected.
\end{abstract}

Keywords: Corruption; Economic growth; Public debt; Unemployment.

\section{INTRODUÇÃO}

A corrupção não é um problema apenas no Brasil. Trata-se de um problema que atinge números elevados no mundo todo. Dados da ONU apontam que aproximadamente 1 trilhão de dólares são liquidados em forma de suborno todos os anos. Enquanto isso, aproximadamente 2,6 trilhões de dólares

\footnotetext{
${ }^{1}$ Graduado em Ciências Econômicas pela Universidade Federal de Mato Grosso. E-mail: ian_vinicius@msn.com

${ }^{2}$ Graduada em Estatística pela Universidade Federal de Mato Grosso. E-mail: alinetatianejung@gmail.com

${ }^{3}$ Doutor em Administração pela UNINOVE, Mestre em Agronegócio e Desenvolvimento Regional e graduado em Ciências Econômicas. Atualmente é professor de Economia na Faculdade de Economia da UFMT. Estuda temas relacionados à ética e religiosidade no contexto econômico. maxmurtinho@ hotmail.com
} 
são desviados por gestão corrupta (JÚNIOR, 2016). No Brasil, estima-se que, só em 2017, aproximadamente 200 bilhões de reais foram desperdiçados com esquemas de corrupção (ISTO É, 2017). Em função disso, serviços essenciais para a população acabam não sendo entregues ou são de péssima qualidade. Pesquisa da Gazeta Online mostra que a cada real desviado pela corrupção, a sociedade é avariada em três reais (SEIXAS, 2017).

Mesmo em países desenvolvidos a corrupção está presente. Na França, por exemplo, 54\% da população francesa considera os políticos como corruptos. Além disso, mais de $80 \%$ da população francesa anseia pela renovação do quadro político por considerar seus políticos corruptos (EURO NEWS, 2017). Nos Estados Unidos (EUA) o auge da corrupção foi chamado de a "Era folheada a ouro", nomeada pelo Mark Twain para definir o que foi um período de inúmeras adversidades sociais disfarçada por uma fina camada de ouro. Homens de grande influência financeira foram apelidados de barões ladrões, por acumular bens, controlar os recursos naturais, remunerar com baixos salários, manipular monopólios ilegais e inflacionar ações de empresas (AQUINO, 2016).

$\mathrm{Na}$ Inglaterra, um ex-ministro britânico, na época diretor do banco HSBC, realizava crimes de evasão fiscal e lavagem de dinheiro do narcotráfico, além de salvaguardar negócios a bancos da Arábia Saudita e Bangladesh com ligação ao financiamento do terrorismo (MONBIOT, 2015). Na Alemanha, um exemplo de caso de corrupção foi o da Siemens, uma das maiores companhias da Tecnologia da Informação do país. Uma investigação apontou que, entre 2001 e 2007, foi gasto em forma de propina aproximadamente US\$ 1,4 bilhão pela empresa, em diversos países, em troca de contratos junto a governos (SÁVIO, 2017). Enfim, todos os países têm quadro de corrupção. O Fórum Econômico Mundial estimou que o custo da corrupção é igual a mais de 5\% do PIB mundial (cerca de US \$2,6 trilhões) (GRAYCAR; SIDEBOTTOM, 2012)

No Brasil, vários são os exemplos de corrupção: desvios de R 42 milhões no Banestado entre 1996 e 2000 no Paraná; o caso "Vampiros da Saúde", tendo sido desviado R\$ 2,4 bilhões entre 1990 e 2004 no Ministério da Saúde; em 1999, o Banco Marka provocou um prejuízo de R \$ 1,8 bilhão aos cofres do Banco Central; desvio de R \$ 923 milhões entre 1992 e 1999 no Tribunal Regional do Trabalho de São Paulo; por fim, a "Máfia dos Fiscais", um desvio de R\$ 18 milhões entre 1998 e 2008 ocorrido na Câmara dos vereadores de São Paulo, envolvendo não só políticos, mas vários servidores públicos concursados (LIMA, 2012). Um dos maiores escândalos de corrupção a nível nacional foi o "Mensalão". Este caso consistiu em repasses de fundos de empresas, que faziam doações ao Partido que ocupava o poder para conquistar o apoio de políticos. O esquema começou em 2002 e só em 2005 foi descoberto, por meio de uma gravação secreta (PEREIRA, 2017).

Quanto a mais recente das operações de investigação, prisão e julgamento de políticos corruptos, a "Operação Lava Jato", tem esse nome inspirado na rede de lavanderias e um posto de combustíveis de Brasília que eram utilizadas como "fachada" das organizações criminosas investigadas para movimentar dinheiro ilícito (PADULA; ALBUQUERQUE, 2018). A Operação Lava Jato é uma operação da Polícia 
Federal que examina esquemas de corrupção envolvendo empresas estatais, diversas empreiteiras e políticos de vários partidos. Teve seu início em 2014, sendo a maior operação contra a corrupção da história do Brasil (POLÍCIA FEDERAL, 2019). Até o presente momento a Operação Lava Jato conseguiu ressarcir aproximadamente $\mathrm{R} \$ 44,4$ bilhões aos cofres públicos (ODILLA, 2018). Em 2019 a operação completou cinco anos, somaram 2.252 anos de condenações para 159 réus, mantendo preso $11 \%$ de todos os 426 denunciados (CERIONI, 2019).

Na pesquisa científica, a corrupção tem duas perspectivas: a corrupção real, que é definida pelo uso de bens públicos para fins privados (POWER; GONZÁLEZ, 2003); e a corrupção percebida, isto é, a percepção da população sobre a corrupção real medida pelo Índice de Percepção da Corrupção - IPC (BOLL, 2010). A corrupção real, entretanto, é uma variável difícil de ser mensurada em função de sua natureza esparsa e não contínua (CARRARO et al., 2016). Com isso, utiliza-se o IPC como proxy da corrupção real. Não deixa de ser um movimento temerário, afinal, o aumento da corrupção percebida pode não se correlacionar com a corrupção real, já que, quanto mais se combate a corrupção, maior é a exposição do tema na mídia, podendo gerar um viés no resultado do IPC. (BOLL, 2010). Mesmo assim, a utilização do IPC está presente nos estudos sobre o tema (POWER; GONZÁLEZ, 2003; BOLL, 2010; CARRARO et al., 2016). Donchev e Ujhelyi (2009) apresentaram evidências de que os índices de corrupção percebida são sensíveis ao aumento na corrupção absoluta (número de casos).

Como a corrupção política implica no uso indevido dos recursos públicos, é razoável supor que contribua para a ineficiência econômica (BOTINHA; LEMES, 2019). Mas, seria a corrupção política capaz de impactar negativamente a economia nacional? Sobre a economia brasileira, é perceptível o baixo desempenho na última década. Por exemplo, houve queda de 3,5\% no PIB de 2015 e de 3,3\% em 2016 (BANCO MUNDIAL, 2019). A taxa de desemprego, atingiu 12,8\% em 2017 (BANCO MUNDIAL, 2019), e, além disso, a morosidade nas reformas estruturais, ao longo da década de 2010, agravou o aumento da relação dívida/PIB. Em função da baixa performance de algumas das variáveis macroeconômicas, verifica-se a formação de um cenário de retração econômica, recessão e crise (FREITAS; JONER, 2018), tendo um efeito perverso no campo social (PEREIRA, 2017).

Freitas e Joner (2018) sustentam que o desempenho ruim dos indicadores econômicos foram consequências de problemas de ordem político-institucional, entre eles, os vários escândalos envolvendo empresas privadas e públicas que revelam o caráter patrimonialista do Governo, ampliando a instabilidade no cenário econômico. Ademais, defronte de indício de vários casos de corrupção, pagamento de propinas e desvios de verbas públicas, o governo revelou-se fraco e sem reações de defesa para enfrentar ou conter a crise que se intensificou diante da expansão dos níveis da dívida pública. Algumas pesquisas demonstraram que a corrupção funcionou como inibidora do crescimento econômico (LAMBSDORFF, 2003; MAURO, 1995; RIVERA-BATIZ, 2002; KAUFMANN; KRAAY, 2003).

Diante disso, este artigo tem como objetivo analisar se há relação de causalidade entre a corrupção brasileira e o crescimento econômico, a dívida pública e o emprego, nos períodos entre 2008 
e 2018. Para isso, foram realizadas análises estatísticas de correlação. A hipótese da pesquisa é a de que o aumento da corrupção impacta negativamente no crescimento econômico e no emprego e contribui positivamente para o aumento da dívida pública.

O artigo está segmentado em seis partes: além desta introdução, na seção 2 é feita a revisão da literatura, na seção 3 são detalhados os procedimentos metodológicos, na seção 4 e 5 são apresentados os resultados e discutidos seus significados. Por fim, as considerações finais, limitações de pesquisa e sugestões de pesquisas futuras.

\section{REVISÃO DA LITERATURA}

A corrupção é um fenômeno abrangente, pois em todos os períodos e lugares que se tem registro, sua ocorrência foi descrita (GOMES, 2010). Pode-se defini-la como o uso de bens públicos para fins privados (POWER; GONZÁLEZ, 2003; ROSE-ACKERMAN, 2004). Trata-se de um conceito de elevada complexidade, de causas diversas, e de difícil esclarecimento, e, por isso, deve ser analisado sob todos os ângulos e de forma multidisciplinar (CORDEIRO, 2017).

Na esfera da gestão, a corrupção pode ser o resultado da adaptação dos agentes, que ao se verem diante de oportunidades que ofereça alguma vantagem, são tentados a agirem de forma antiética face a limitação das instituições fiscalizadoras. Conforme a impunidade se mantém, a confiança na impunidade se torna uma salvaguarda e um "convite" a transgressão (NOGUEIRA; BATISTA, 2018). Assim, os agentes envolvidos perpetuam uma cultura política inata, que delega a corrupção à dimensão da sociedade em geral, atribuindo ao Poder Judiciário toda a responsabilidade para solucionar o problema da corrupção no Brasil, uma vez que os "controles internos são frágeis" (CORDEIRO, 2017). Neste caso tem-se a chamada "corrupção sistêmica". Tal modalidade de corrupção tem caráter mais estrutural pois resulta na estabilização das expectativas normativas dificultando uma aplicação mais consistente do direito e interferindo na constitucionalização e sua legalidade (NOGUEIRA; BATISTA, 2018).

A literatura sobre o tema, além de escassa, tem apresentado resultados inconclusivos (CARRARO, et al., 2016). Encontrou-se, por exemplo alguma evidência de relação direta entre corrupção e criação (PALIFKA, 2006; MELO; SAMPAIO; OLIVEIRA, 2015). Também evidenciou-se relação negativa entre corrupção e empreendedorismo (BOLOGNA; ROSS, 2015).

Ainda no tema do empreendedorismo, Dutta, Kar e Roy (2011) sugerem que a presença de uma estrutura burocrática corrupta pode arruinar o ímpeto empreendedor, de modo que, a corrupção influencia na realocação de talentos empreendedores para outra atividade menos produtiva. Mas, Dreher e Gassebner (2013), numa amostra de 43 países, encontraram evidências de que a corrupção aumenta a atividade empreendedora. Diante da aparente contradição de resultados, Carraro et al. (2016) levanta a questão da existência de dois argumentos praticamente opostos na relação entre corrupção e empreendedorismo. 
Enquanto autores como Huntington (1968) e Leff (1964) defendem que a corrupção incentiva a criação de novas empresas e estimula a atividade empreendedora, outros como Baumol (1990), afirmam que a corrupção nada mais é que uma despesa econômica que eleva os custos de transações e aumenta a incerteza dos ganhos. Pesquisadores nesta linha, como Acemoglu e Verdier (1998) sugerem que quando a corrupção é maior, mais trabalhadores optam por trabalhar no setor público. Esse resultado é conhecido como má alocação de talentos (CARRARO et al., 2016).

A pesquisa seminal sobre corrupção e economia é a de Mauro (1995). O autor fez análises transversais para estimar os efeitos da corrupção sobre o crescimento econômico, tendo encontrado relação inversa entre corrupção e crescimento. Os estudos de Lambsdorff (2003), Rivera-Batiz (2002) e Kaufmann e Kraay (2003) também denunciam a corrupção como inibidora do crescimento econômico. Já Tanzi e Davoodi (1998) estudaram o comportamento do investimento público diante da corrupção. Concluíram que a corrupção pode reduzir o crescimento por aumentar o investimento público e, ao mesmo tempo, reduzir sua produtividade. Al-Marhubi (2000), ao pesquisar a relação entre corrupção e inflação, encontrou uma associação positiva significativa.

Akçay (2006) analisou o impacto da corrupção sobre o Índice de Desenvolvimento Humano de 63 países, tendo concluído haver relação negativa entre índices de corrupção e o desenvolvimento humano. Ainda sobre desenvolvimento, Cordeiro (2017) sugere que é inevitável que o desenvolvimento econômico dos países não esteja, em certa medida, conectado à corrupção sistêmica e institucionalizada dos atores envolvidos na gestão do Estado.

O autor também sustenta que a corrupção pode ter uma relação direta com a desigualdade de renda do país. Isso ocorre porque, quando a corrupção está institucionalizada, uma parte dos recursos são aplicados em subornos para obter licenças, acesso preferencial a mercados, limitando a sua capacidade para investir em aumentos de produtividade e em inovação e/ou não sendo necessário devido ao monopólio ou oligopólio gerado pela aliança corrupta entre o governo e as empresas inseridas no esquema (CASTRO, 2007).

Um exemplo disso é a corrosão na imagem e valor de mercado da Petrobras, depois das revelações da Lava Jato dos esquemas de corrupção. Ficou evidente a alocação ineficiente de recursos resultando numa maior concentração da riqueza nas mãos de empresários e políticos (HOPE, 2017). Tendo isso em vista, a próxima seção tratará dos procedimentos metodológicos da pesquisa.

\section{PROCEDIMENTOS METODOLÓGICOS}

O presente estudo é de caráter quantitativo. Para alcançar os propósitos do estudo, buscou-se identificar: os dados dos indicadores macroeconômicos de crescimento, dívida pública e emprego; dados do Índice de Percepção da Corrupção; dados do cadastro de contas julgadas irregulares pelo Tribunal de Contas da União. Todos os dados foram coletados para o decênio entre 2008 e 2018. 
Os dados econômicos foram coletados do Banco Mundial e do Banco Central do Brasil. Uma vez com os dados, os gráficos foram construídos por meio da ferramenta Excel. O procedimento foi basicamente estatístico. Utilizou-se para análise as seguintes ferramentas: Verificação de Normalidade; Coeficiente de Correlação de Pearson; c) Coeficiente Tau de Kendall. Para a verificação de normalidade utilizou-se o teste de Shapiro-Wilk. Este teste tem sido um dos mais utilizados para teste de normalidade, devidos às suas poderosas propriedades em comparação a outros testes (ROYSTON, 1982). No teste, define-se na hipótese nula que os dados seguem uma distribuição normal. Com isso, se o valor-p for menor que o nível de significância (usualmente 5\%), rejeita-se a hipótese nula.

Quanto ao Coeficiente de Correlação de Pearson (r), mede-se o grau da correlação linear entre duas variáveis quantitativas. É um índice adimensional com valores situados ente -1 e 1 para representar a intensidade de uma relação linear entre dois conjuntos de dados. Quanto mais próximo de 1, maior a correlação positiva entre duas variáveis, quanto mais próximo de -1, maior a correlação negativa. Quanto mais próximo de zero, menor a evidência de correlação entre duas variáveis (ARAUJO; SANTOS; GOMES; 2019). Já o Coeficiente Tau de Kendall Taub ( $\pi$ ou $t_{b}$ ), apresenta propriedades matemáticas que o tornam mais robusto a dados extremos (outliers), com maior capacidade de inferência populacional e menor erro de estimativa (MIOT, 2019), tornando-a uma excelente ferramenta complementar de análise estatística.

Para proxy de corrupção utilizou-se o Índice de Percepção de Corrupção e o cadastro de contas julgadas irregulares pelo Tribunal de Contas da União (TCU). O IPC baseia-se em fontes de dados recolhidos ao longo dos últimos 24 meses à sua apuração e é analisado por instituições independentes especializadas em governança e ambiente de negócios, que atuam com parceiros no governo, empresas e sociedade civil, visando aperfeiçoar as medidas para o combate à corrupção. Isso se deve ao fato de a instituição gestora do índice Transparency Internacional ser responsável também pelos projetos da Convenção Anticorrupção da OCDE (Organização para a Cooperação e Desenvolvimento Econômico), Convenção das Nações Unidas contra a Corrupção e Pacto Global da ONU, ou seja, ela trabalha em parceria com grandes organizações mundiais (BOTINHA; LEMES, 2019).

Quanto aos dados do cadastro de contas julgadas irregulares pelo Tribunal de Contas da União. As atribuições do TCU estão previstas no artigo nº 71 da Constituição de 1988. Essas incumbências, somadas às inspeções e auditorias, fazem com que o TCU atue de maneira preventiva, inibindo a prática de irregularidades (BOLL, 2010). O intuito da utilização dos dados do TCU é não depender apenas da evolução do IPC para analisar a evolução da corrupção no Brasil, já que o dado é tão criticado pelo risco de não se correlacionar com a corrupção real. O TCU emite uma lista com os gestores que possuíram contas julgadas irregulares em um cadastro chamado Cadastro de Responsáveis com Contas Julgadas Irregulares Cadirreg. Nele estão as pessoas, físicas ou jurídicas, que possuíram ou não cargos eletivos /função pública, vivas ou falecidas. É com as informações do cadastro que os dados são enviados, em anos eleitorais, à Justiça Eleitoral, incumbida de decretar a inelegibilidade de um gestor (SOUZA, 2019). 
Quanto aos dados econômicos, a evolução do PIB anual foi a medida de crescimento econômico utilizada. Quanto à dívida pública, o motivo de sua presença na análise foi pelo fato de que uma elevação pode significar ineficiência na gestão dos recursos públicos. Trata-se de uma importante medida macroeconômica, pois permite fazer comparações internacionais de forma mais eficaz (AMORIM, 2019; GOBETTI; SCHETTINI, 2010). Para a análise, utilizou-se os dados da dívida bruta e líquida. Por fim, a presença da taxa de desemprego se deve a sua importância e impacto para o cidadão.

\section{DISCUSSÃO E RESULTADOS}

Nesta seção são apresentados os dados do IPC, Produto Interno Bruto (PIB), taxa de desemprego e dívida pública, bem como os resultados dos cálculos estatísticos realizados. Pode-se observar na Tabela 1 que o Índice de Percepção da Corrupção do Brasil no decênio oscilou entre 35 e 43 pontos. Nota-se também uma elevação da pontuação no início do decênio (entre 2008 e 2012), todavia nos últimos dois anos (2017 e 2018) a pontuação caiu sensivelmente. Disso resulta uma forte queda na posição do Brasil no ranking mundial, onde, uma vez tendo alcançado a $69^{\mathrm{a}}$ posição por três vezes $(2010$, 2012 e 2014), termina o decênio na $105^{\text {a }}$ posição, a pior desde que o índice começou a ser calculado. Para a Transparência Internacional, o agravamento da percepção da corrupção nos últimos anos coincide com o escândalo da Lava a Jato, que tornou exposta a corrupção sistêmica (G1, 2019). A instituição que elabora o ranking avalia 180 países.

Quanto ao número de processos julgados irregulares pelo Tribunal de Contas da União, houve aumento constante no início do decênio (de 2008 a 2010), e, após uma queda em 2011 (1366 casos irregulares), o número de processos irregulares volta a subir chegando ao pico de 2198 processos irregulares no ano de 2016. Nos dois anos finais do decênio evidencia-se leve queda, mas mantendo-se um número maior de 2000 processos (Tabela 1). Comparando-se os dados do IPC com os do TCU, percebe-se que os picos e vales dos dados não se coincidem. Ainda assim, pode-se inferir que, apesar de todos os esforços das instituições fiscalizadoras, a corrupção brasileira persiste se mantendo em altos patamares, principalmente nos últimos três anos do período em análise.

Tabela 1 - Índice de Corrupção Percebida e posição do Brasil no ranking da Transparência Internacional $-2008-18$

\begin{tabular}{lccccccccccc}
\hline Discriminação & $\mathbf{2 0 0 8}$ & $\mathbf{2 0 0 9}$ & $\mathbf{2 0 1 0}$ & $\mathbf{2 0 1 1}$ & $\mathbf{2 0 1 2}$ & $\mathbf{2 0 1 3}$ & $\mathbf{2 0 1 4}$ & $\mathbf{2 0 1 5}$ & $\mathbf{2 0 1 6}$ & $\mathbf{2 0 1 7}$ & $\mathbf{2 0 1 8}$ \\
\hline Índice TI & 35 & 37 & 37 & 38 & 43 & 42 & 43 & 38 & 40 & 37 & 35 \\
\hline Posição TI & 80 & 75 & 69 & 73 & 69 & 72 & 69 & 76 & 79 & 96 & 105 \\
\hline $\begin{array}{l}\mathbf{N}^{\mathbf{0}} \text { processos Irregulares } \\
\text { TCU }\end{array}$ & 1497 & 1520 & 1940 & 1833 & 1366 & 1409 & 1592 & 2002 & 2198 & 2116 & 2034 \\
\hline $\begin{array}{l}\text { Fonte: Transparência Internacional (2019) e Tribunal de Contas da União (2019). } \\
\text { na }\end{array}$
\end{tabular}


A Tabela 2 apresenta a evolução do PIB e da taxa de desemprego entre os períodos de 2008 e 2018. Chama atenção o fato de que, a partir de 2014, a economia brasileira não teve mais o mesmo desempenho do início da década de 2010. Além de um crescimento muito baixo, de 0,5\% em 2014, os dois anos seguintes, 2015 e 2016, foram de crescimento negativo. Nos dois anos que encerram o decênio, 2017 e 2018, apresentaram crescimentos muito baixos, pouco maior que 1\%. Supõem-se que a Nova Matriz Econômica (NME), implantada a partir de 2011 tenha diminuído o ímpeto produtivo da economia brasileira (BARBOSA FILHO, 2017).

Quanto a taxa de desemprego, no decênio em análise, oscilava entre 6\% e $8 \%$ até 2015, quando começou a subir sensivelmente, indo para 11,6\% em 2016, 12,8\% em 2017 e 12,5\% em 2018 (Tabela 2). A incompatibilidade entre o avanço da demanda e oferta de trabalhadores disponíveis no interior do mercado de trabalho revela o aumento recente do desemprego no Brasil metropolitano, cuja determinação encontra-se associada à crise na economia nacional (POCHMANN, 2015).

Tabela 2 - Crescimento do PIB e taxa de desemprego (2008 - 2018)

\begin{tabular}{lccccccccccc}
\hline Discriminação & $\mathbf{2 0 0 8}$ & $\mathbf{2 0 0 9}$ & $\mathbf{2 0 1 0}$ & $\mathbf{2 0 1 1}$ & $\mathbf{2 0 1 2}$ & $\mathbf{2 0 1 3}$ & $\mathbf{2 0 1 4}$ & $\mathbf{2 0 1 5}$ & $\mathbf{2 0 1 6}$ & $\mathbf{2 0 1 7}$ & $\mathbf{2 0 1 8}$ \\
\hline $\begin{array}{l}\text { Produto Interno } \\
\text { Bruto (\%) }\end{array}$ & 5,09 & $-0,12$ & 7,53 & 3,97 & 1,92 & 3,00 & 0,50 & $-3,54$ & $-3,30$ & 1,06 & 1,11 \\
\hline $\begin{array}{l}\text { Taxa de } \\
\text { Desemprego (\%) }\end{array}$ & 7,3 & 8,5 & 7,7 & 6,9 & 7,2 & 6,9 & 6,7 & 8,4 & 11,6 & 12,8 & 12,5 \\
\hline
\end{tabular}

Fonte: Banco Mundial (2019).

Tanto o PIB quanto o emprego evoluíram negativamente nos últimos anos. Sicsú (2019) afirma que isto poder estar relacionado com uma tênue tendência de queda nos investimentos. Segundo o autor, houve queda de quase $2 \%$ no $3^{\circ}$ e $4^{\circ}$ trimestre de 2013. Em 2014, continuou a retração dos níveis de investimentos e de utilização da capacidade instalada, houve recessão no $2^{\circ}$ trimestre do ano e, logo, a quantidade de trabalhadores com carteira assinada no setor privado começou a cair a partir do $3^{\circ}$ trimestre.

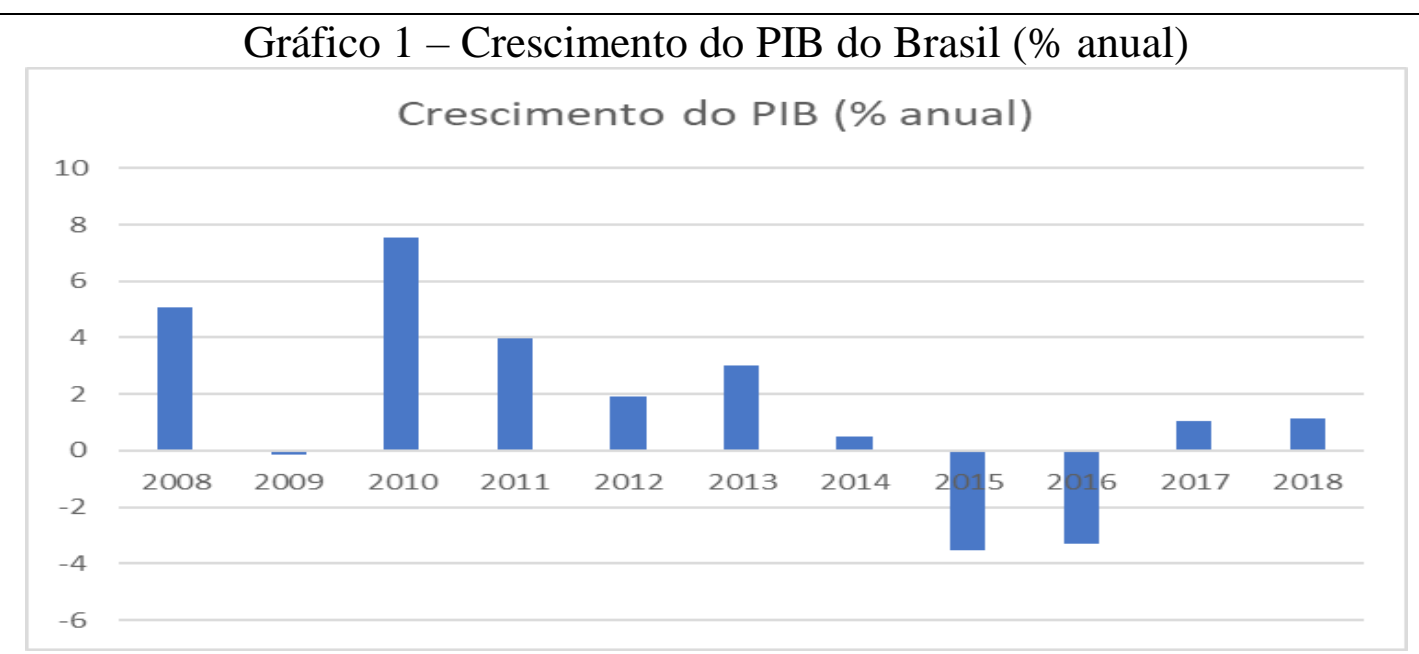

Fonte: Banco Mundial (2019). 


\section{Gráfico 2 - Desemprego Total Percentual Da População Ativa Total do Brasil}

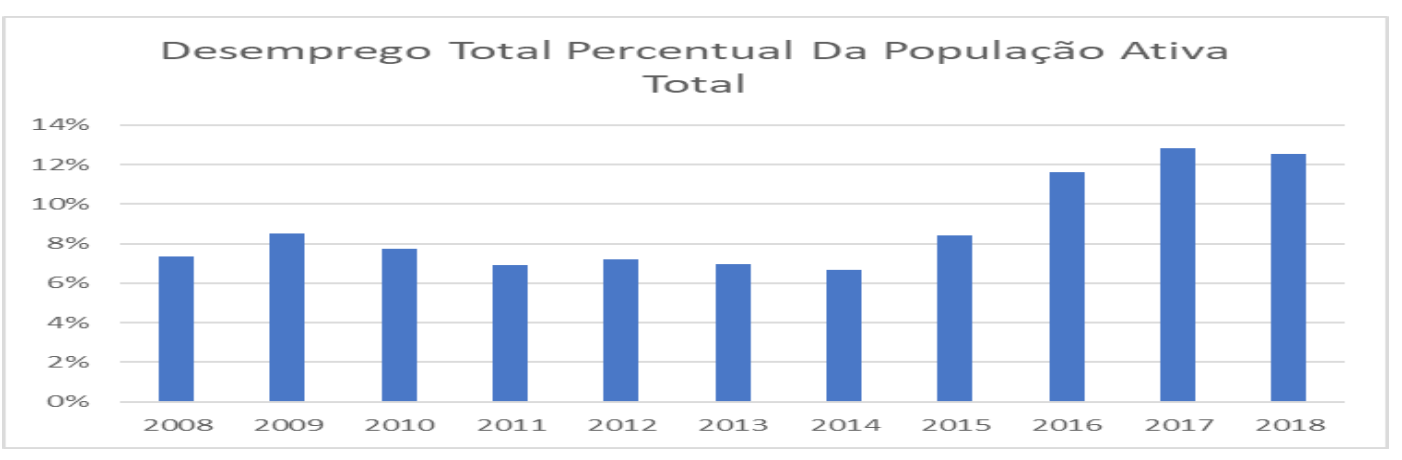

Fonte: Banco Mundial (2019).

Em relação aos ajustes fiscais do Brasil, ocorrem basicamente relacionados a supressão em investimentos e elevação de cargas tributárias, medidas de políticas contracionistas. De forma que essas medidas vêm de um longo histórico pós ditadura e persistem até o ano de 2017. Fundamentada na proposta de orçamento anual levada ao congresso, a discussão fiscal é feita com sustentação nos dados da Dívida Bruta do Governo Geral (DBGG) e Dívida Líquida do Governo Geral (DLGG), sendo distintas as duas dívidas (AMORIM, 2019).

Na Tabela 3 evidencia-se um forte crescimento nas DLGG e DBGG. A gestão das contas públicas vem a vários anos permitindo que a dívida fique cada vez maior, como podemos observar no Gráfico 3. Segundo o Relatório do Tesouro Nacional temos duas situações futuras a primeira é estabilização da DBGG/PIB um pouco acima de $80 \%$, nesse cenário todas as reformas estruturais foram implantadas de forma integral e, estima-se que a DBGG/PIB tenderia a trajetória decrescente a partir de 2022. A segunda situação e a ausência de reformas que resultaria em um cenário caótico no qual DBGG/PIB passaria de 90\% (TESOURO NACIONAL, 2018). O crescimento do percentual da DLGG/PIB e da DBGG/PIB é extremamente alarmante, há um crescimento desordenado a partir de 2014 chegando a alcançar em novembro de 2017, 74,4\% do PIB conforme o Gráfico 4.

Tabela 3 - Dívida Líquida e Bruta Do Governo Geral em valores (R\$ milhões) e percentual do PIB

\begin{tabular}{|c|c|c|c|c|}
\hline Descrição & DLGG & DBGG & DLGG (\% PIB) & DBGG (\% PIB) \\
\hline 2008/Jan & $\mathrm{R} \$ 1.173 .193$ & $\mathrm{R} \$ 1.579 .474$ & 42,7 & 57,5 \\
\hline 2008/Jul & $\mathrm{R} \$ 1.258 .613$ & $\mathrm{R} \$ 1.632 .714$ & 42,8 & 55,5 \\
\hline 2009/Jan & $\mathrm{R} \$ 1.192 .857$ & $\mathrm{R} \$ 1.775 .483$ & 38,2 & 56,9 \\
\hline 2009/Jul & $\mathrm{R} \$ 1.309 .625$ & $\mathrm{R} \$ 1.908 .617$ & 41,0 & 59,7 \\
\hline 2010/Jan & $\mathrm{R} \$ 1.354 .994$ & $\mathrm{R} \$ 2.015 .577$ & 40,2 & 59,8 \\
\hline 2010/Jul & $\mathrm{R} \$ 1.446 .567$ & $\mathrm{R} \$ 2.027 .615$ & 39,6 & 55,5 \\
\hline 2011/Jan & $\mathrm{R} \$ 1.496 .047$ & $\mathrm{R} \$ 2.058 .854$ & 38,1 & 52,4 \\
\hline 2011/Jul & $\mathrm{R} \$ 1.573 .965$ & $\mathrm{R} \$ 2.204 .281$ & 37,5 & 52,5 \\
\hline 2012/Jan & $\mathrm{R} \$ 1.571 .757$ & $\mathrm{R} \$ 2.287 .084$ & 35,6 & 51,9 \\
\hline 2012/Jul & $\mathrm{R} \$ 1.534 .757$ & $\mathrm{R} \$ 2.480 .228$ & 33,2 & 53,6 \\
\hline 2013/Jan & $\mathrm{R} \$ 1.599 .521$ & $\mathrm{R} \$ 2.623 .366$ & 32,9 & 54,0 \\
\hline 2013/Jul & $\mathrm{R} \$ 1.614 .286$ & $\mathrm{R} \$ 2.744 .023$ & 31,6 & 53,7 \\
\hline
\end{tabular}




\begin{tabular}{ccccc}
\hline 2014/Jan & $\mathrm{R} \$ 1.650 .951$ & $\mathrm{R} \$ 2.829 .599$ & 30,7 & 52,6 \\
\hline 2014/Jul & $\mathrm{R} \$ 1.799 .679$ & $\mathrm{R} \$ 2.983 .108$ & 32,1 & 53,2 \\
\hline 2015/Jan & $\mathrm{R} \$ 1.934 .269$ & $\mathrm{R} \$ 3.315 .324$ & 33,4 & 57,2 \\
\hline 2015/Jul & $\mathrm{R} \$ 2.043 .299$ & $\mathrm{R} \$ 3.684 .951$ & 34,5 & 62,2 \\
\hline 2016/Jan & $\mathrm{R} \$ 2.286 .788$ & $\mathrm{R} \$ 3.992 .779$ & 38,1 & 66,5 \\
\hline 2016/Jul & $\mathrm{R} \$ 2.672 .668$ & $\mathrm{R} \$ 4.213 .993$ & 43,5 & 68,7 \\
\hline 2017/Jan & $\mathrm{R} \$ 3.047 .148$ & $\mathrm{R} \$ 4.399 .047$ & 48,3 & 69,8 \\
\hline 2017/Jul & $\mathrm{R} \$ 3.323 .550$ & $\mathrm{R} \$ 4.722 .126$ & 51,6 & 73,3 \\
\hline 2018/Jan & $\mathrm{R} \$ 3.532 .846$ & $\mathrm{R} \$ 4.904 .275$ & 53,7 & 74,5 \\
\hline 2018/Jul & $\mathrm{R} \$ 3.641 .522$ & $\mathrm{R} \$ 5.186 .540$ & 54,3 & 77,4 \\
\hline
\end{tabular}

Fonte: Banco Central do Brasil (2019).

Gráfico 3 - Dívida Líquida E Bruta Do Governo Geral do Brasil (R\$ milhões)

\section{Dívida Líquida e Bruta do Governo Geral}

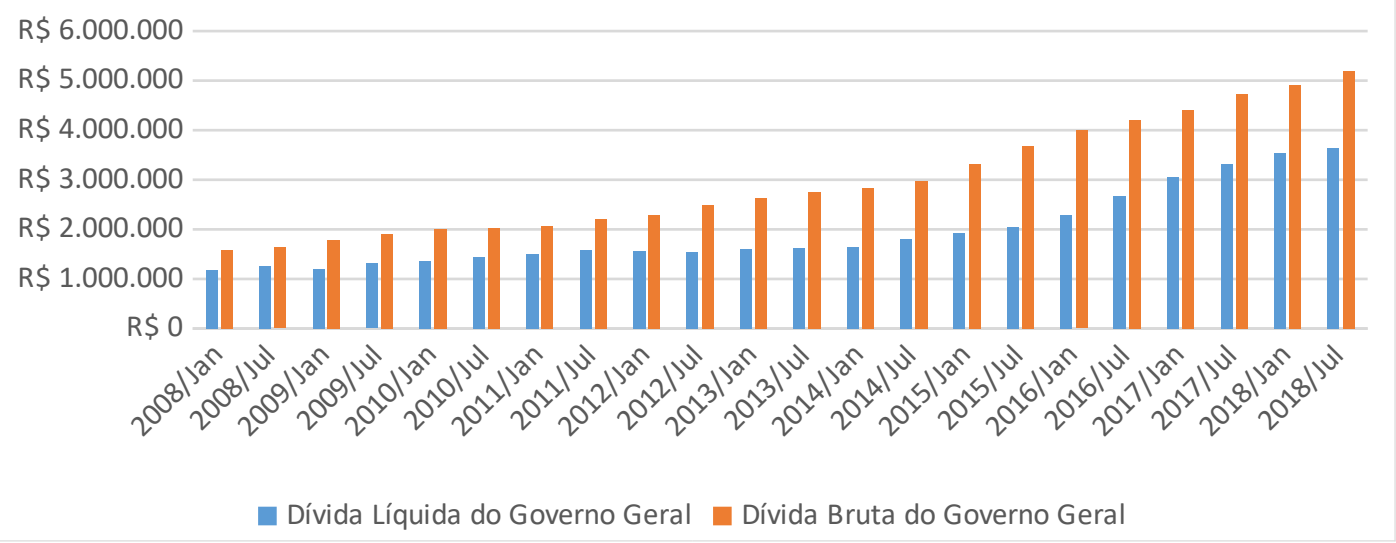

Fonte: Banco Central do Brasil (2019).

Gráfico 4 - Dívida líquida e bruta do governo geral (\% PIB)

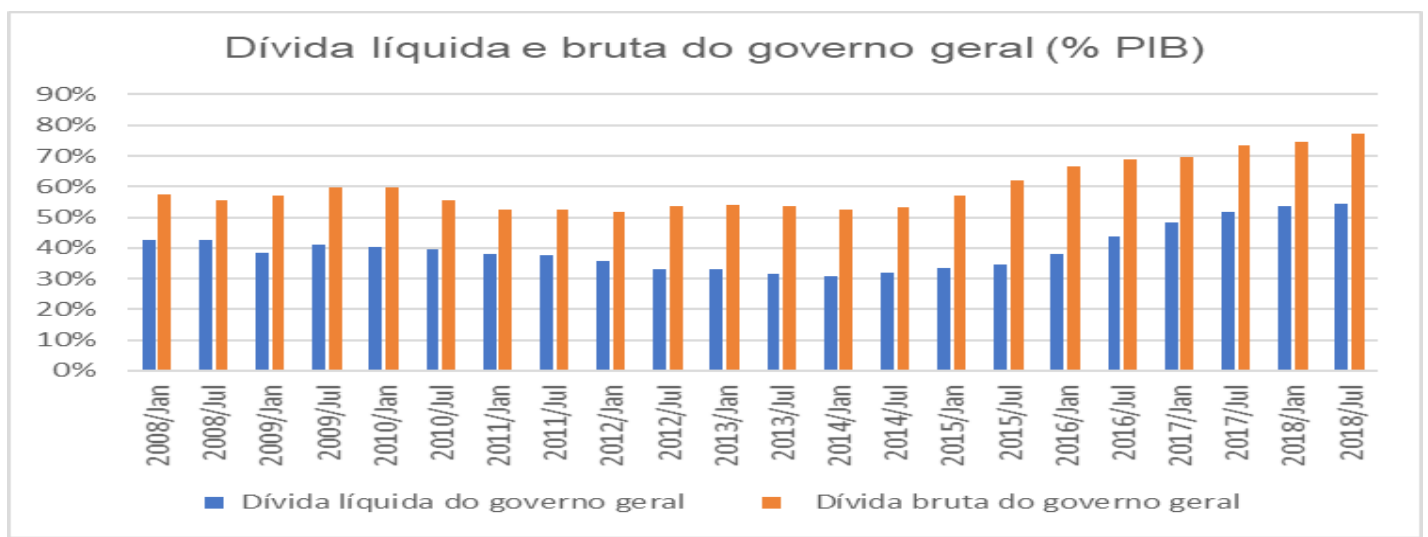

Fonte: Banco Central do Brasil (2019).

Antes de calcular a correlação entre as variáveis de corrupção e de economia é preciso verificar a normalidade dos dados. Por meio de um teste de normalidade é possível observar se as variáveis possuem distribuição normal. Caso as variáveis não apresentem distribuição normal, os testes de 
correlação não devem ser paramétricos. O teste de normalidade escolhido foi o Shapiro-Wilk (Tabela 7). Verifica-se que os valor-p das variáveis IPC, TCU, PIB e DBGG foram, respectivamente, 0,127, 0,235, 0,862 e 0,248. Esses valores são maiores que o nível de significância de 5\%, ou seja, são variáveis que apresentam distribuição normal. Já as variáveis DLGG e DESEMPREGO apresentaram o valor-p de 0,033 e 0,006, respectivamente. São valores menores que o nível de significância, ou seja, não possuem distribuição normal. Outra modalidade de teste será necessária para essas variáveis.

Tabela 7 - Resultados dos testes de normalidade Shapiro-Wilk

\begin{tabular}{ccccccc}
\hline Variáveis & IPC & TCU & PIB & DBGG & DLGG & Desemprego \\
Valor-p & 0,127 & 0,235 & 0,862 & 0,248 & 0,033 & 0,006 \\
\hline
\end{tabular}

Fonte: Elaboração própria (2019)

Para as variáveis IPC, TCU, PIB e DBGG, que possuem distribuição normal calculou-se a correlação por meio do Coeficiente de Pearson. Os resultados para as correlações IPC/PIB e IPC/DBGG foram, respectivamente, de -0,21 e -0,08 (Tabela 8). Quanto mais próximos de 1 ou -1, maior a evidência de correlação entre as variáveis, seja positiva ou negativa. Entretanto, como pode-se observar, os resultados são muito próximos a zero, o que denota não haver correlação entre as variáveis. Já os resultados das correlações TCU/PIB e TCU/DBGG foram, respectivamente $-0,33$ e 0,7 , denotando correlação fraca no primeiro caso e correlação moderada no segundo. Para o teste de correlação entre o IPC e as variáveis sem distribuição normal, DLGG e Desemprego, empreendeu-se cálculo por meio do Coeficiente de Kendall Tau-b. Todavia, os resultados encontrados, 0,09 para a relação IPC/DLGG e 0,40 para IPC/Desemprego (Tabela 8) evidenciam a mesma situação das variáveis anteriores, ou seja, os valores encontrados muito próximos de zero, o que significa que não há evidência de correlação entre as variáveis. O mesmo teste empreendido para as variáveis TCU/DLGG e TCU/DES foram ambos de 0,5 , demonstrando haver correlação fraca em ambas as relações.

Tabela 8 - Resultados dos testes de correlação

\begin{tabular}{|c|c|c|c|c|c|c|c|c|}
\hline Discriminação & IPC e PIB & $\begin{array}{l}\text { IPC e } \\
\text { DBGG }\end{array}$ & $\begin{array}{c}\text { TCU e } \\
\text { PIB }\end{array}$ & $\begin{array}{l}\text { TCU e } \\
\text { DBGG }\end{array}$ & $\begin{array}{l}\text { IPC e } \\
\text { DLGG }\end{array}$ & $\begin{array}{l}\text { IPC e } \\
\text { DES }\end{array}$ & $\begin{array}{l}\text { TCU e } \\
\text { DLGG }\end{array}$ & $\begin{array}{c}\text { TCU e } \\
\text { DES }\end{array}$ \\
\hline $\begin{array}{l}\text { Correlação de } \\
\text { Pearson (r) }\end{array}$ & $-0,21$ & $-0,08$ & $-0,33$ & 0,7 & & & & \\
\hline $\begin{array}{c}\text { Coeficiente tau } \\
\text { de Kendall }\end{array}$ & & & & & 0,09 & $-0,40$ & 0,5 & 0,5 \\
\hline
\end{tabular}

Fonte: Autoria própria (2019).

Também realizou-se o Teste de Correlação de Pearson (r) para as variáveis que representam a medida de corrupção, IPC e TCU. O valor-p encontrando foi de 0,42 , ou seja, surpreendentemente, as variáveis que medem a corrupção do país possuem fraca correlação. Com isso, é possível afirmar que 
as análises estatísticas apresentaram resultados que não confirmam a hipótese de que haja relação de causalidade entre a corrupção e as variáveis econômicas.

\section{CONSIDERAÇÕES FINAIS}

Este artigo teve como objetivo analisar a influência da corrupção brasileira sobre o crescimento econômico, a dívida pública e o emprego, nos períodos entre 2008 e 2018. Dentre as esparsas pesquisas sobre corrupção na economia, tem sido comum os resultados não serem significativos o suficiente para confirmar as hipóteses de pesquisa. A escolha de três variáveis econômicas, a evolução do Produto Interno Bruto e da Dívida Pública e a taxa de desemprego, foi necessária a fim de delimitar as variáveis econômicas que fossem próximas de alguma forma à gestão ineficiente por motivos de corrupção, possibilitando assim observar se haveria relação de causalidade. A escolha do crescimento econômico e da dívida pública, se devem ao fato de que o Brasil, segundo Carraro et al. (2016) apresenta crescimento econômico limitado em função do elevado gasto público proporcionado pelo seu endividamento.

Além disso, para os autores, a corrupção já está enraizada no processo de negócios entre a sociedade e o governo (CARRARO et al., 2016). Quanto ao emprego, trata-se de uma das principais variáveis econômicas para o cidadão, supõem-se um potencial para influenciar na percepção do cidadão sobre possíveis variáveis negativas que contribuísse de algum modo no aumento do desemprego.

Como o objetivo do artigo era verificar uma relação de causalidade entre corrupção e economia, foram realizados alguns testes estatísticos de correlação. A hipótese foi a de que o aumento da corrupção influenciaria na deterioração do crescimento e emprego e contribuiria para o aumento da dívida pública. Os testes, no entanto, não apresentaram resultados robustos o suficiente para manter a ideia de causalidade entre corrupção e economia no Brasil. Isso não significa, é claro, que a corrupção não afete de alguma forma a economia do país. Mas ajuda a evidenciar a fragilidade dos dados disponíveis para a análise da corrupção. Power e González (2003) esclarecem que o Índice de Percepção da Corrupção é uma medida de reputação, não um indicador concreto de corrupção. Um indicador com maior embasamento empírico seria preferível, mas um indicador dessa natureza ainda é muito difícil, talvez impossível de ser construída em função da natureza desviante da variável corrupção.

Apesar das dificuldades, ao menos, a abordagem feita nesta pesquisa permite encarar a relação entre economia e corrupção. Tal procedimento, ainda é pouco realizado no Brasil. Novos estudos se fazem necessários para contribuir com a pesquisa sobre corrupção e economia no Brasil. Vale ressaltar que os resultados encontrados estão sujeitos ao questionamento metodológico. Carraro et al. (2016) afirmam que para se chegar a um consenso sobre o impacto causal da corrupção no empreendedorismo, muitos artigos são precisos, principalmente com diferentes metodologias e banco de dados. Empreender esforços no levantamento de novos dados que possam servir de proxy para a corrupção real deve ser também um caminho importante para futuras pesquisas sobre corrupção e economia. 


\section{REFERÊNCIAS}

ACEMOGLU, D.; VERDIER, T. Property rights, corruption and the allocation of talent: a general equilibrium approach. Economic Journal, St Andrews, v. 108, n. 450, p. 1381-1403, 1998.

AKÇAY, S. Corruption and human development. Cato Journal, Washington, DC, v. 26, n. 1, p. 2948, 2006.

AL-MARHUBI, F. Corruption and inflation. Economics Letter, [S.1.], v. 66, n. 2, p. 199-202, 2000.

AMORIM, S. Dívida Pública: Interação entre dívida bruta e dívida líquida do governo federal. In: Revista Razão Contábil \& Finanças, v. 9, n. 2, 2019.

AQUINO, R. de. A corrupção nos EUA. Época, [S.I.], 27/10/2016. Disponível em: $<$ https://epoca.globo.com/colunas-e-blogs/ruth-de-aquino/noticia/2013/08/corrupcaonos-eua-vivendoe-aprendendo.html $>$. Acesso em: 08/09/2019.

ARAUJO, J. V. G. A.; SANTOS, M. dos; GOMES, C. F. S. Desenvolvimento de um código em Python para geração de Matrizes de Correlação de Pearson com laços a partir de "n" variáveis tomadas duas a duas, 2019.

BANCO MUNDIAL. Desemprego, total (\% da força de trabalho total) (estimativa modelada da OIT). Disponível em: <https://datos.bancomundial.org/indicador/SL.UEM.TOTL.ZS?locations=BR>. Acesso em: 11/10/2019.

BANCO MUNDIAL. Crescimento do PIB (\% anual) - Brasil. Disponível em: <https://datos.bancomundial.org/indicador/NY.GDP.MKTP.KD.ZG?locations=BR>. Acesso em: $11 / 10 / 2019$.

BANCO MUNDIAL. PIB per capita (US \$ atual) - Brasil. Disponível em: <https://data.worldbank.org/indicator/NY.GDP.PCAP.CD?locations=BR>. Acesso em: 11/10/2019.

BARBOSA FILHO, F. de H. A crise econômica de 2014/2017. Estudos Avançados, v. 31, n. 89, p. 51-60, 2017.

BAUMOL, W. Entrepreneurship: productive, unproductive and destructive. Journal of Political Economy, Chicago, IL, v. 98, n. 5, p. 893-921, 1990.

BOLL, J. L. S. A corrupção governamental no Brasil: construção de indicadores e análise da sua incidência relativa nos estados brasileiros. Dissertação (Mestrado em Economia do Desenvolvimento) - Pontifícia Universidade Católica do Rio Grande do Sul, Porto Alegre, 2010.

BOLOGNA, J.; ROSS, A. Corruption and entrepreneurship: evidence from Brazilian municipalities. Public Choice, [S.1.], v. 165, n. 1, p. 59-77, 2015.

BOTINHA, R. A.; LEMES, S. Corrupção percebida: Uma análise da associação com o ambiente contábil dos países do G20. Advances in Scientific and Applied Accounting, v. 1, n. 1, p. 120-139, 2019.

CASTRO, C. Corrupção e crescimento econômico - uma relação não linear na união europeia dos 15 ? In: Revista de Direito e Gestão Fiscal, 33, jan. - mar., p. 111-124, 2007. 
CARRARO, A.; RIBEIRO, F. G.; COSTA, G. W.; MENEZES, G. R.; CANEVER, M. D.; FERNANDEZ, R. N. Does governamental corruption affect entrepreneurship in Brazil? In: Ensaios FEE, Porto Alegre, v. 37, n. 3, p. 615-642, 2016.

CERIONI, C. A operação já colecionou 2.252 anos de condenações para 159 réus e mantém $11 \%$ de seus 426 denunciados na cadeia. Exame, [S.I.], 18/03/2019. Disponível em:<https://exame.abril.com.br/brasil/cinco-anos-da-lava-jato-os-resultados-edesafios-da-operacao/>. Acesso em: 11/10/2019.

CORDEIRO, C. P.B. S. A corrupção sob um prisma histórico-sociológico: uma análise de suas principais causas e efeitos. Revista Eletrônica Direito e Conhecimento, v. 1, n. 2, 2017.

DONCHEV, D.; UJHELYI, G. What Do Corruption Indices Measure?. Social Science Electronic Publishing, p. 1-45, aug. 2009. Disponível em: <http://papers.ssrn.com/ sol3/papers.cfm?abstract_id=1124066>. Acesso em: 16/12/2019.

DREHER, A.; GASSEBNER, M. Greasing the wheels? The impact of regulations and corruption on firm entry. Public Choice, [S.1.], v. 155, n. 3, p. 413-432, 2013.

DUTTA, N.; KAR, S.; ROY, S. Informal sector and corruption: an empirical investigation for India. Bonn: IZA, 2011. (IZA Discussion paper, n.5579). Retrieved from <http://ftp.iza.org/dp5579.pdf> on 1 July 2014.

EURO NEWS. Corrupção: $O$ "cancro" na política francesa a que poucos escapam. França, 07/02/2017. Disponível em: <https://pt.euronews.com/2017/02/07/corrupcao-o-cancro-na-politicafrancesa-a-que-poucos-escapam>. Acesso em: 04/06/2019.

FREITAS, G. S.; JONER, H. A economia brasileira no início do século XXI: Um olhar estendido até a crise de 2015. in: Revista de Desenvolvimento Econômico, v. 2, n. 40, 2018.

G1. Entenda Operação Lava Jato da polícia federal. São Paulo, 14/04/2014. Disponível em: <http://g1.globo.com/politica/noticia/2014/04/entenda-operacao-lavajato-da-policia-federal.html>. Acesso em: 02/11/2019.

GOBETTI, S. W.; SCHETTINI, B. P. Dívida Líquida e Dívida Bruta: uma abordagem integrada para analisar a trajetória eo custo do endividamento brasileiro. Texto para Discussão, Instituto de Pesquisa Econômica Aplicada (IPEA), 2010.

GOMES, J. V. L. A corrupção em perspectivas teóricas. Teoria e Cultura, v. 5, n. 1 e 2, 2010.

GRAYCAR, A.; SIDEBOTTOM, A. Corruption and control: a corruption reduction approach. Journal of Financial Crime, v.19, n.4, p. 384-399, 2012.

HOPE, K. R. Fighting corruption in developing countries: Some aspects of policy from lessons from the field. Journal of Public Affairs, v. 17, n. 4, 2017.

HUNTINGTON, S. P. (Ed.) Political order in changing societies. New Haven: Yale University Press, 1968.

ISTO É. Brasil perde cerca de R $\mathbf{2 0 0}$ bilhões por ano com corrupção, diz MPF. [S.I.], 07/02/2017. Disponível em:<https://istoe.com.br/brasil-perde-cerca-de-r-200bilhoes-por-ano-comcorrupcao-diz-mpf/>. Acesso em: 10/10/2019.

JÚNIOR, E. US\$ 2,6 trilhões são roubados por meio de corrupção todos os anos. 
ONU NEWS, New York, 09/12/2016. Disponível em: <https://news.un.org/pt/story/ 2016/12/1571041-us-26-trilhoes-sao-roubados-por-meio-de-corrupcao-todos-osanos>. Acesso em: $02 / 05 / 2019$.

KAUFMANN, D.; KRAAY, A. Governance and growth: which causes which. Washington, D.C.: World Bank Institute and the Development Research Group, 2003. (The World Bank Working Papers).

LAMBSDORFF, G. J. How corruption affects productivity. Kyklos, Malden, MA, v. 56, n. 4, p. $143-$ 156, 2003.

LEFF, N. H. Economic development through bureaucratic corruption. American Behavioral Scientist, [S.1.], v. 8, n. 3, p. 8-14, 1964.

LIMA, C. Os maiores escândalos de corrupção do Brasil. Super Interessante, 24/02/2012. Disponível em: <https://super.abril.com.br/mundo-estranho/os-maioresescandalos-de-corrupcao-do-brasil/>. Acesso em: 25/09/2019.

MAURO, P. Corruption and growth. Quarterly Journal of Economics, Cambridge, MA, v. 110, n. 3, p. 681-712, 1995.

MELO, F. B. de; SAMPAIO, L.; OLIVEIRA, R. Corrupção burocrática e empreendedorismo: uma análise empírica dos estados brasileiros. in: Revista de Administração Contemporânea, Rio de Janeiro, v. 19, n. 3, p. 374-397, 2015.

MONBIOT, G. Londres, a Meca dos corruptos. Trad. Vila Vudu. Carta Maior, 28/03/2015. Disponível em: <https://www.cartamaior.com.br/?/Editoria/Pelo-Mundo/ Londres-a-Meca-doscorruptos/6/33152>. Acesso em: 15/12/2019.

NOGUEIRA, P. B.; BATISTA, C. Implicações da Legislação Simbólica no Sistema Jurídico. in: Revista Brasileira de Teoria Constitucional, v. 3, n. 1, p. 1-15, 2018.

ODILLA, F. Lava Jato: MPF recupera R $\$ 11,9$ bi com acordos, mas devolver todo dinheiro às vítimas pode levar décadas. BBC Brasil, Londres, 17/03/2018. Disponível em: <https://www.bbc.com/portuguese/brasil-43432053>. Acesso em: 02/08/2019.

PADULA, A. J. A.; ALBUQUERQUE, P. H. M. Corrupção governamental no mercado de capitais: Um estudo acerca da operação Lava Jato. in: Revista de Administração de Empresas, v. 58, n. 4, p. 405-417, 2018.

PALIFKA, B. Corruption and entrepreneurship in Brazil. 2006. Paper presented at the 150-mile conference, Edinburg, Texas, 2006.

PEREIRA, J. M. Avaliação dos efeitos da crise econômica-política-ética nas finanças públicas do Brasil. in: Revista Ambiente Contábil, v. 9, n. 2, p. 117-141, 2017.

POCHMANN, M. Ajuste econômico e desemprego recente no Brasil metropolitano. Estudos avançados, v. 29, n. 85, p. 7-19, 2015.

POLÍCIA FEDERAL. Operação Lava Jato. [S.I.]. Disponível em: <http://www.pf.gov.br/imprensa/lava-jato>. Acesso em: 14/08/2019. 
POWER, T.; GONZALEZ, J. Cultura política, capital social e percepções sobre corrupção: Uma investigação quantitativa em nível mundial. in: Revista de Sociologia e Política. n. 21, nov. 2003, pp. 51-69.

RIVERA-BATIZ, F. L. Democracy, governance, and economic growth: theory and evidence. Review of Development Economics, Malden ,MA, v. 6, n. 2, p. 225-247, 2002.

ROSE-ACKERMAN, S. The challenge of poor governance and corruption. Copenhagen: Copenhagen Consensus, 2004.

ROYSTON, J. P. Algorithm AS 181: the W test for normality. Journal of the Royal Statistical Society: série C-apllied statistics, London, v. 31, n. 2, p. 176180, 1982.

SÁVIO, G. Combate a corrupção: O exemplo que vem da Alemanha. Diário de Pernambuco, Pernambuco, 19/12/2017. Disponível em:

<https://www.diariodepernambuco.com.br/noticia/politica/2017/12/combate-a-corrupcao-o-xemploque-vem-daalemanha.html>. Acesso em: 20/10/2019.

SEIXAS, B. Saiba qual é o preço da corrupção no Brasil. Gazeta Online, [S.I.], 29/05/2017.

Disponível em: <https://www.gazetaonline.com.br/noticias/economia/ 2017/05/saiba-qual-e-o-precoda-corrupcao-no-brasil-1014059906.html>. Acesso em: 10/11/2019.

SICSÚ, J. A economia da depressão está de volta? in: Revista Economia \& Sociedade [S.I.], 2019.

SOUZA, T. C. S. C. Padrões de condenações do Tribunal de Contas da União a gestores públicos e participação política. Dissertação de Mestrado. Universidade Federal de Campina Grande, 2019.

TANZI, V.; DAVOODI, H. (Ed.). Roads to nowhere: how corruption in public investment hurts growth. Washington, DC: International Monetary Fund, 1998. (Economic Issues, 12).

TESOURO NACIONAL. Relatório Quadrimestral de Projeções da Dívida Pública 2018.

Disponível em:

<http://www.tesouro.gov.br/documents/10180/318974/RelatorioProje\%C3\%A7oesDivida/ac957aaa84db-4e66-9ad8-0042c291e45d 2017>. Acesso em: 11/10/2019.

TRANSPARÊNCIA INTERNACIONAL. Índice de Percepção da Corrupção 2018. Disponível em: <https://ipc2018.transparenciainternacional.org.br>. Acesso em: 11/10/2019.

Recebido: $16 / 05 / 2020$

Aceito: 23/09/2020

Publicado: outubro de 2020. 